\title{
Correction to: LncRNA MATN1-AS1 for Prediction of Prognosis in Osteosarcoma Patients and Its Cellular Function
}

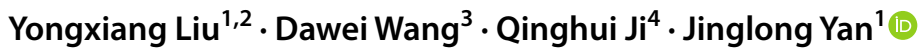

Accepted: 4 October 2021 / Published online: 13 October 2021

○) Springer Science+Business Media, LLC, part of Springer Nature 2021

\section{Correction to: Molecular Biotechnology https://doi.org/10.1007/s12033-021-00394-9}

Unfortunately in the original publication, the affiliations associated with the author Yongxiang Liu were incomplete. Also the order of affiliations should be changed. The original article has been corrected.

The correct list of affiliations is given in this erratum.
Publisher's Note Springer Nature remains neutral with regard to jurisdictional claims in published maps and institutional affiliations.

The original article can be found online at https://doi.org/10.1007/ s12033-021-00394-9.

Jinglong Yan

JinglongYan2nd@163.com

1 Department of Sixth Orthopedics, The 2nd Affiliated Hospital of Harbin Medical University, Harbin 150000, China

2 Department of Orthopedics, Yantai City Yantaishan Hospital, Yantai 264000, China

3 Department of Orthopedics, Zhangjiakou First Hospital, Zhangjiakou 075000, China

4 Department of Orthopedics, The First Affiliated Hospital of Jiamusi University, Jiamusi 154000, China 